\title{
A COMPARATIVE STUDY OF BONE MARROW ASPIRATION AND BONE MARROW BIOPSY IN HEMATOLOGICAL DISEASES
}

\author{
Nirajan Mainali, Neha Homagai, Pratap Sagar Tiwari, Arun Giri
}

\begin{abstract}
:
Introduction: Bone marrow examination is an important diagnostic tool to evaluate various disorder including both neoplastic and non-neoplastic hematological diseases. The two most important techniques used for the diagnosis are bone marrow aspiration and bone marrow trephine biopsy which are complementary to each other.
\end{abstract}

Aim and objectives: To compare the diagnostic value of bone marrow aspiration and biopsy.

Material and methods: A total of 88cases with both bone marrow aspiration and biopsy were included in the study. All the aspirate smears were routinely stained by Jenner Giemsa while the trephine biopsy sections were stained by routine Hematoxylin and Eosin stain.. All the smears and sections were reviewed and the findings on BMA and BMB were compared and the final correlation done.

Result: Hypolastic marrow was the most common diagnosis followed by immune thrombocytopenic purpura (ITP). But the diagnostic accuracy of ITP on aspiration was $100 \%$ in compare to hypoplastic marrow $(81.25 \%)$. The diagnosis accuracy of BMA in our study was $84.09 \%$.

Conclusion: Bone marrow aspiration and biopsy complement each other. Bone marrow aspiration provides better study of the cell, whereas biopsy provides detail about the pattern of cellular distribution, hence when performed together it gives better diagnostic accuracy.

Key Words: Bone marrow aspiration, Bone marrow biopsy, Hypoplastic marrow, leukemia.

\section{INTRODUCTION:}

Bone marrow examination is an important diagnostic tool to evaluate various disorder including both neoplastic and non-Neoplastic hematological diseases. The bone marrow evaluation may either confirm clinically suspected disease or may provide the previously unsuspected diagnosis. $(1,2)$

The two most important techniques used for the diagnosis are bone marrow aspiration and bone marrow trephine biopsy which are complementary to each other. Aspiration of the marrow is primarily utilized for cytological assessment with analysis directed towards morphology and obtaining a differential cell count. Biopsy is essential for diagnosis in a dry tap or blood tap which occurs when the marrow is fibrotic or densely cellular. Only a biopsy allows a complete assessment of marrow architecture and pattern of distribution of any abnormal infiltrates (3). The present study was conducted to compare the role of bone marrow aspirate cytology and trephine biopsy to formulate an effective and 
rapid method for diagnosing wide spectrum of hematological diseases.

\section{AIM AND OBJECTIVES:}

To compare the diagnostic value of bone marrow aspiration and biopsy.

\section{MATERIALS AND METHODS:}

This was a two year retrospective study done in the department of pathology, Nobel medical college hospital and research centre, from march 2013 to February 2015. A total of 140 cases presented with clinical hematological disorders; of which only 88 were biopsied and the correlation done. The bone marrow samples were obtained from the posterior iliac spine. The aspiration needle was passed perpendicular into the cavity of the bone and $0.3-0.6 \mathrm{ml}$ of marrow content was sucked with the help of a $10 \mathrm{ml}$ Syringe.

The trephine biopsy was performed using Jamshidi needle with the length of the biopsy core ranging from 0.8 to $1.5 \mathrm{~cm}$. The biopsy was then fixed for minimum of 24 hours in $10 \%$ buffered formalin and then decalcified overnight picric acid. The fixation of the biopsy core was followed by automated tissue processing, paraffin embedding and sectioning. All the aspirate smears were routinely stained by Jenner Giemsa while the trephine biopsy sections were stained by routine Hematoxylin and Eosin stain.. All the smears and sections were reviewed and the findings on BMA and BMB were compared and the final correlation done.

\section{RESULT:}

A total of 88 cases were subjected to bone marrow aspiration and bone marrow biopsy. Hypolastic marrow was the most common diagnosis followed by idiopathic thrombocytopenic purpura in bone marrow aspiration. Hypoplastic marrow was the most common diagnosis in the bone marrow biopsy also but the number of cases were more in compare to aspiration. $2^{\text {nd }}$ most common diagnosis was ITP which diagnosis accuracy was $100 \%$ when compared with bone marrow aspiration.

Total of six (6) cases came out to be dry while doing bone marrow aspiration. Among with Hypoplastic marrow was the most common case (4/6). One case each of leukemia and myelodysplastic disorder was also encountered.

The overall diagnostic accuracy of bone marrow aspiration cytology in diagnosing hematological disorders was $84.09 \%$.

\begin{tabular}{|c|c|c|c|}
\hline Disorders & BMA & BMB & $\begin{array}{l}\text { Diagnostic } \\
\text { accuracy }(\%)\end{array}$ \\
\hline $\begin{array}{l}\text { Megaloblastic } \\
\text { anemia }\end{array}$ & 12 & 10 & \\
\hline $\begin{array}{l}\text { Micronormoblastic } \\
\text { anemia }\end{array}$ & 10 & 9 & \\
\hline Hypoplastic anemia & 26 & 32 & 81.25 \\
\hline Leukemia & 4 & 5 & 80.00 \\
\hline $\begin{array}{l}\text { NHL-infiltration on } \\
\text { bone marrow }\end{array}$ & 1 & 1 & 100 \\
\hline $\begin{array}{l}\text { Myelodysplastic } \\
\text { syndrome }\end{array}$ & 3 & 5 & 60.00 \\
\hline Multiple myeloma & 2 & 2 & 100.0 \\
\hline $\begin{array}{l}\text { Immune thrombo- } \\
\text { cytopenic purpura }\end{array}$ & 15 & 15 & 100.0 \\
\hline Metastasis & 2 & 3 & 66.67 \\
\hline Leishmaniasis & 3 & 3 & 100.00 \\
\hline Dry tap & 6 & - & \\
\hline Normal & 4 & 3 & \\
\hline
\end{tabular}

Table I: Comparative evaluation of bone marrow aspiration and bone marrow biopsy diagnosis

\begin{tabular}{|l|l|}
\hline Cases & Biopsy diagnosis \\
\hline Hypoplastic anemia & 4 \\
\hline Leukemia & 1 \\
\hline Myelodysplastic syndrome & 1 \\
\hline
\end{tabular}

Table II: BMTB diagnosed cases in dry taps on BMA

\section{DISCUSSION:}

Bone marrow examination is an important investigation carried out in the routine practice for the diagnosis of various hematological and non-hematological disorders. BMTB is a relatively safe procedure with adverse event reported in only 
.12 to $.34 \%$ of procedures. The commonest complication of BMTB was mild hemorrhage.(4)Major risk factor for hemorrhage was MPD and increased bleeding in a case of polycythaemia vera(5). The commonest hematological disorder in the present study was hypoplastic marrow with 32 cases belonging to this subset. In the study done by parajuli et al (6) megaloblastic anemia was the most common diagnosis while the study done by Vidisha Mahajan et al (7) nutritional anemia was the most common diagnosis. The diagnosis accuracy of BMA in our study was $84.09 \%$ which was similar to the study done by parajuli et al (6). Diagnosis accuracy of BMA was slightly lower in the study done by Smita Chandra et al (77.5\%) (8) Our study showed trephine biopsy was also superior to BMA in diagnosing hypoplastic/ aplastic anemia similar to study by parajuli et al(4) and Gupta $\mathrm{N}$ et al. (9) We encountered a single case of NHL infiltrating the bone marrow which was diagnosed both on BMA and BMB, where as in the study done by vidisha et al (7) much of the cases could not be diagnose on the BMA alone, which stressed out point that both aspiration and biopsy should be done in case of suspected NHL.(7). 2 out of $3(66 \%)$ Metastatic carcinoma were diagnosed on bone marrow aspiration which are as similar to the study done by Smita Chandra (8). 33\% of metastatic carcinoma was only found to be positive in BMA in the study done by Donald et al (10) To conclude, Bone marrow aspiration and biopsy complement each other. It is always a clinician decision to choose whether to perform a Bone marrow aspiration or a biopsy or both. Better result is yielded out when both are perform simultaneously. Bone marrow aspiration provides better study of the cell, whereas biopsy provides detail about the pattern of cellular distribution, cellularity, infiltration by tumor cells and provides a block if immunohistochemistry is to perform later on.

\section{References:}

1. Chandra S, Chandra H, Saini S. Bone marrow metastasis by solid tumors - probable hematological indicators and comparison of bone marrow aspirate, touch imprint and trephine biopsy. Hematology 2010; 15:368-72.

2. Nanda A, Basu S, Marwaha N. Bone marrow trephine biopsy as an adjunct to bone marrow aspiration. J Assoc Physicians India 2002; 50:893-5.

3. Bain BJ, Clark DM, Wilkins BS. The normal bone marrow. In Bain BJ, Clark DM \& Wilkins BS editors. Bone marrow Pathology 4th Ed. Singapore, Wiley- black well, 2010:1-51.

4. Khatun $\mathbf{H}$ et al, Bone marrow trephine biopsy in hematological diseases: A study of 53 cases, J Dhaka Med Coll. 2013; 22(2): 207-210.

5. Malempati S, Joshi S, Lai S, Braner DAV, Tegtmeyer K. Bone marrow aspiration and biopsy. N Engl J Med 2009; 361(15): 28.

6. Parajuli S, Tuladhar A. Correlation of bone marrow aspiration and biopsy findings in diagnosing hematological disorders - a study of 89 cases Journal of Pathology of Nepal (2014) Vol. 4, 534-538.

7. Mahajan $\mathbf{V}, \mathbf{K}$ aushal $\mathbf{V}$ et al A comparative study of bone marrow aspiration and bone marrow biopsy in hematological and non-hematological disorders An institutional experience JIACM 2013; 14(2): 133-5

8. Smita Chandra and Harish Chandra. Comparison of bone marrow aspirate cytology, touch imprint cytology and trephine biopsy for bone marrow evaluation. Hematol Rep 2011; 19:3-22.

9. Nitin Gupta, Ram Kumar, Arvind Khajuria. Diagnostic assessment of bone marrow aspiration smears, touch imprints and trephine biopsy in hematological disorders. JK Science 2010; 12:130-3.

10. Donald P, Chikappa G. Comparative evaluation of bone marrow aspirate particle smears biopsy imprints and biopsy sections. Am J Hematology 1986; 22:381-9.

Correspondence Address: Dr. Nirajan Mainali, Lecturer, Nobel Medical College, E-mail: mainali_nirajan@hotmail.com 